\title{
Weaning from veno-arterial extra-corporeal membrane oxygenation: which strategy to use?
}

\author{
Sofia Ortuno ${ }^{1}$, Clément Delmas ${ }^{2}$, Jean-Luc Diehl ${ }^{1}$, Clotilde Bailleul ${ }^{1}$, Aymeric Lancelot ${ }^{1}$, Mahassen Naili ${ }^{3}$, \\ Bernard Cholley ${ }^{4}$, Romain Pirracchio ${ }^{4}$, Nadia Aissaoui ${ }^{1,5}$ \\ ${ }^{1}$ Department of Intensive Care Cnit, Hôpital Européen Georges Pompidou (HEGP) Assistance Publique-Hôpitaux de Paris (APHP) and Université \\ Paris Descartes, Paris, France; ${ }^{2}$ Intensive Cardiac Care Unit, Rangueil University, Toulouse, France; ${ }^{3}$ Department of Cardiology, Hôpital Européen \\ Georges Pompidou, APHP, Paris, France; ${ }^{4}$ Department of Anesthesiology and Intensive Care, Paris, Hôpital Européen Georges Pompidou, APHP, \\ Université Paris-Descartes, Paris, France; ${ }^{5}$ Paris-Cardiovascular-Research-Center, INSERM U970, Paris, France \\ Correspondence to: Nadia Aissaoui, MD, PhD. Department of Intensive Care, Hôpital Européen Georges Pompidou, 20 rue Leblanc, 75015 Paris, \\ France. Email: nadia.aissaoui@ aphp.fr.
}

Refractory cardiogenic shock patients may be rescued by veno-arterial extracorporeal membrane oxygenation (VA ECMO). After a few days of mechanical assistance, the device can sometimes be successfully removed if the patient has partially or fully recovered from the condition that required the use of ECMO. The percentage of patients with refractory cardiogenic shock who are successfully weaned from ECMO varies from $31 \%$ to $76 \%$. Weaning does not mean survival, because $20 \%$ to $65 \%$ of patients weaned from VA ECMO support do not survive to hospital discharge. The high death rate after successful weaning shows that many questions remain unresolved in this field. In this review, we will discuss the various factors influencing survival and a successful weaning from VA ECMO, in addition to weaning approaches proposed in the literature. Based on this information, we will propose a strategy to optimize the weaning process.

Keywords: Veno-arterial extracorporeal membrane oxygenation (VA ECMO); weaning; echocardiography

Submitted Jun 29, 2018. Accepted for publication Aug 13, 2018.

doi: $10.21037 /$ acs.2018.08.05

View this article at: http://dx.doi.org/10.21037/acs.2018.08.05

\section{Introduction}

Veno-arterial extracorporeal membrane oxygenation (VA ECMO) may be used to rescue patients with refractory cardiogenic shock (1-10). The main indication for VA ECMO may be medical cardiogenic shock, including that associated with acute myocardial infarction, fulminant myocarditis, acute exacerbation of severe chronic heart failure, drug intoxication, hypothermia and acute circulatory failure due to intractable arrhythmia (1-7). VA ECMO may also be used for patients with post-cardiotomy cardiac failure or after cardiac or pulmonary transplantation, or cardiac arrest requiring cardiopulmonary resuscitation $(1,4,8)$. VA ECMO may be used in some particular situations for patients with pulmonary embolism, sepsis associated cardiomyopathy and pulmonary hypertension (1-10).

VA ECMO is used as a bridge to myocardial recovery and cardiac transplantation. It may also be used as "a bridge to a bridge", that is, before implantation of a ventricular assist device. No randomized controlled trials have compared VA ECMO to other mechanical support systems in patients with cardiogenic shock. However, several nonrandomized studies suggest that the early use of ECMO offers a survival advantage in such circumstances (1-12).

After a few days of mechanical assistance, the device can sometimes be successfully removed if the patient has partially or fully recovered from the condition that required the use of ECMO. However, to date, only a few studies have reported strategies for weaning from VA ECMO (13-16). The percentage of patients with refractory cardiogenic shock who are successfully weaned from ECMO varies from $31 \%$ to $76 \%$, depending on the underlying cause of cardiogenic shock and the definition of successful weaning (10-16). Indeed, the definition of successful weaning varies in the reported studies. Some consider weaning successful if 
the patient survives for longer than 48 hours after ECMO explantation (6-8). We defined patients successfully weaned from VA ECMO as those having ECMO removed and not requiring further mechanical support because of recurring cardiogenic shock over the following 30 days $(13,16)$. This last definition seems to be the most clinically relevant.

Moreover, weaning does not mean survival, because $20 \%$ to $65 \%$ of patients weaned from VA ECMO support do not survive to hospital discharge. In one recent database study assessing 5,263 patients from Japan, more than $50 \%$ of patients weaned off ECMO died in hospital (12). Conversely, the high rate of ECMO-related complications may prompt removal of the device as soon as possible (7). This manuscript will discuss the various factors influencing survival and a successful weaning from VA ECMO, in addition to weaning approaches proposed in the existing literature. Based on this information, we will propose a strategy to optimize the weaning process.

\section{Factors predicting death in weaned patients}

As reported, successful weaning from ECMO does not mean patient survival. Several studies have assessed the predictors of death after ECMO weaning mainly in the setting of post-cardiotomy shock and out-of-hospital cardiac arrest (6,9-11). Interestingly, markers associated with death after weaning were diabetes, obesity, door-to- VA ECMO implantation time, cardiopulmonary resuscitation duration, poor renal and liver function, high lactate levels, and high SOFA score $(6,9-11,17)$.

\section{Which factors can help to predict successful weaning from VA ECMO?}

Few studies aimed at identifying criteria to predict which patients can be successfully weaned from ECMO, using the same methodology (Table 1) (13-15,18-20). They assessed prospectively and daily clinical, biological and/or echo parameters until death, weaning or bridge to long-term assist-device or heart transplantation. These studies were only observational and parameters were compared between weaned and non-weaned patients. The main limitations of these studies were their observational design and the number of the patients $(2,3)$.

\section{Clinical predictors: pulsed pressure}

Among clinical parameters, neither age, sex, co-morbidities, or severity at the time of VA ECMO implantation were identified as predictors of successful weaning. Pulse pressure appeared to be the only clinical factor associated with weaning success $(13,15,21)$. In our study assessing 51 patients, among the 38 patients undergoing a decrease of ECMO flow, mean pulse pressure was higher in the successfully weaned group $52 \pm 12$ vs. $39 \pm 15 \mathrm{mmHg}$ (5) in others. Our result was confirmed by Pappalardo et al., who reported a mean pulse pressure of 59 [53-69] $\mathrm{mmHg}$ in the 49 successfully weaned patients. However, no clinically relevant predictive threshold could be identified (15).

\section{Biomarkers}

Cardiac and vascular biomarkers were shown to be associated with outcomes in patients with heart failure (20). Reflecting on pathological processes happening in the heart, may be useful to predict cardiac recovery and to help to identify VA ECMO patients who would recover. Luyt et al. examined whether biomarkers could predict cardiac recovery in 41 patients receiving VA ECMO (20). They analyzed circulating concentrations of the $\mathrm{N}$-terminal fragment of the B-type natriuretic peptide, troponin Ic, the midregional fragment of the proatrial natriuretic peptide, proadrenomedullin and copeptin on the first day of ECMO and at day 3 , and 7 . There was no difference in the absolute values of these biomarkers or in their kinetics during the first week between patients who were weaned from ECMO and those who were not.

A few studies suggested that lactate and lactate clearance could help for ECMO weaning (22). Li et al. evaluated lactate at baseline, within 6 hours and 12 hours, in 123 patients under VA-ECMO for a refractory postcardiotomy cardiogenic shock. Mean lactate concentration (OR 1.1; 95\% CI, 1.0-1.2; $\mathrm{P}<0.001$ in the 6-hour model; and OR $1.2 ; 95 \% \mathrm{CI}, 1.1-1.4 . \mathrm{P}<0.001$ in the 12 -hour model) and lactate clearance (OR 0.5; 95\% CI, 0.3-0.9; $\mathrm{P}=0.001$ in the 6-hour model and OR 0.1; 95\% CI, 0.02-0.3; $\mathrm{P}=0.001$ in the 12 -hour model) were significantly associated with hospital mortality and unsuccessful weaning (22).

\section{Echo parameters}

In cardiogenic shock (CS) patients assisted by VA ECMO, echographic cardiac assessment is of paramount importance to set the degree of extracorporeal blood flow and concomitant supports such as mechanical ventilation, fluid loading and inotropic therapy $(13,15,23,24)$. 


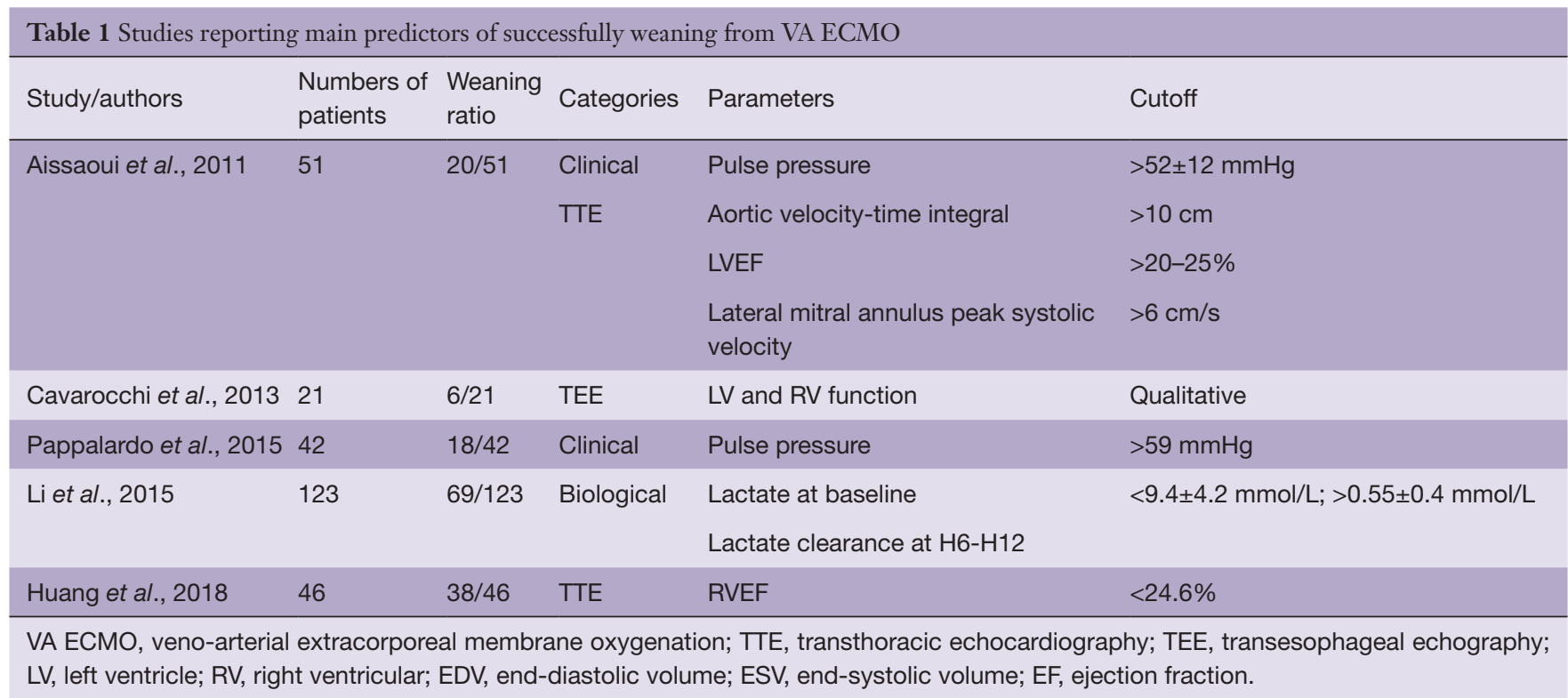

As comprehensive and dynamic echocardiographic measurements are mandatory to manage patients with CS patients assisted by ECMO, it seemed logical to assess the ability of echo parameters to predict successful weaning $(23,24)$. Thus, we assessed the ability of echocardiographic variables to predict successful weaning in 51 patients receiving VA ECMO (13). High values of parameters assessing systolic LV function, such as aortic velocitytime integral, LVEF and lateral mitral annulus peak systolic velocity were associated with successful weaning. The Doppler parameters reflecting LV filling pressures (i.e., mitral $\mathrm{E}$ and TDI Ea velocities and $\mathrm{E} / \mathrm{Ea}$ ) did not differ significantly at each ECMO flow level and were not predictive of weaning outcome, suggesting that preload conditions were comparable for the two groups (13).

Precise RV assessment, except RV dilation, has rarely been investigated. Interestingly, in a recent study assessing 46 patients, successfully weaned patients had smaller RV dimensions, better RVEF, higher RV area change and magnitudes of RV strain (18). The value of tricuspid annular plane systolic excursion and the severity of tricuspid regurgitation did not differ between the two groups. Of note, 3D-RV EF has the highest area under the curve $(0.90$, $\mathrm{P}<0.001$ ) for weaning success, with a cutoff value at $24.6 \%$ in this series (18).

Thus, current data suggest that echocardiography is an important tool to determine both the recovery of $\mathrm{LV}$ and $\mathrm{RV}$ function (Figure 1) and the readiness of patients for weaning from ECMO support, whereas early measurements of cardiac biomarkers are not useful for identifying those who will recover (Table 1).

\section{Hemodynamic assessment during the weaning attempt}

Hemodynamic assessment may be useful during the weaning trial in teams using the tool for critically ill patient monitoring. The pulmonary arterial catheter measurements give important information regarding the preloads of $\mathrm{RV}$ and LV. For patients to be considered for VA ECMO weaning, hemodynamic variables with the pump off should be as follows: cardiac index $>2.4 \mathrm{~L} / \mathrm{min} / \mathrm{m}^{2}$, mean blood pressure $>60 \mathrm{mmHg}$, pulmonary capillary wedge pressure $<18 \mathrm{~mm} \mathrm{Hg}$ and central venous pressure $<18 \mathrm{mmHg}$ (25).

\section{Microcirculation parameters}

Akin et al. recently reported in a study on 13 patients that the assessment of the sublingual microcirculation functional parameters, such as the total vessel density and perfused vessel density, may be of worth alongside weaning from VA ECMO (19). Patients who were not weaned from VA ECMO had clear deterioration in total small and large vessel densities. In contrast, in weaned patients, there was no or minimal alterations in microcirculation. A comparison of the microcirculatory parameters with echocardiographic parameters values showed good correlation. Such measurements may be attractive and complementary but are not available in many centers; their reproducibility is questionable and they are sensitive to artefacts 

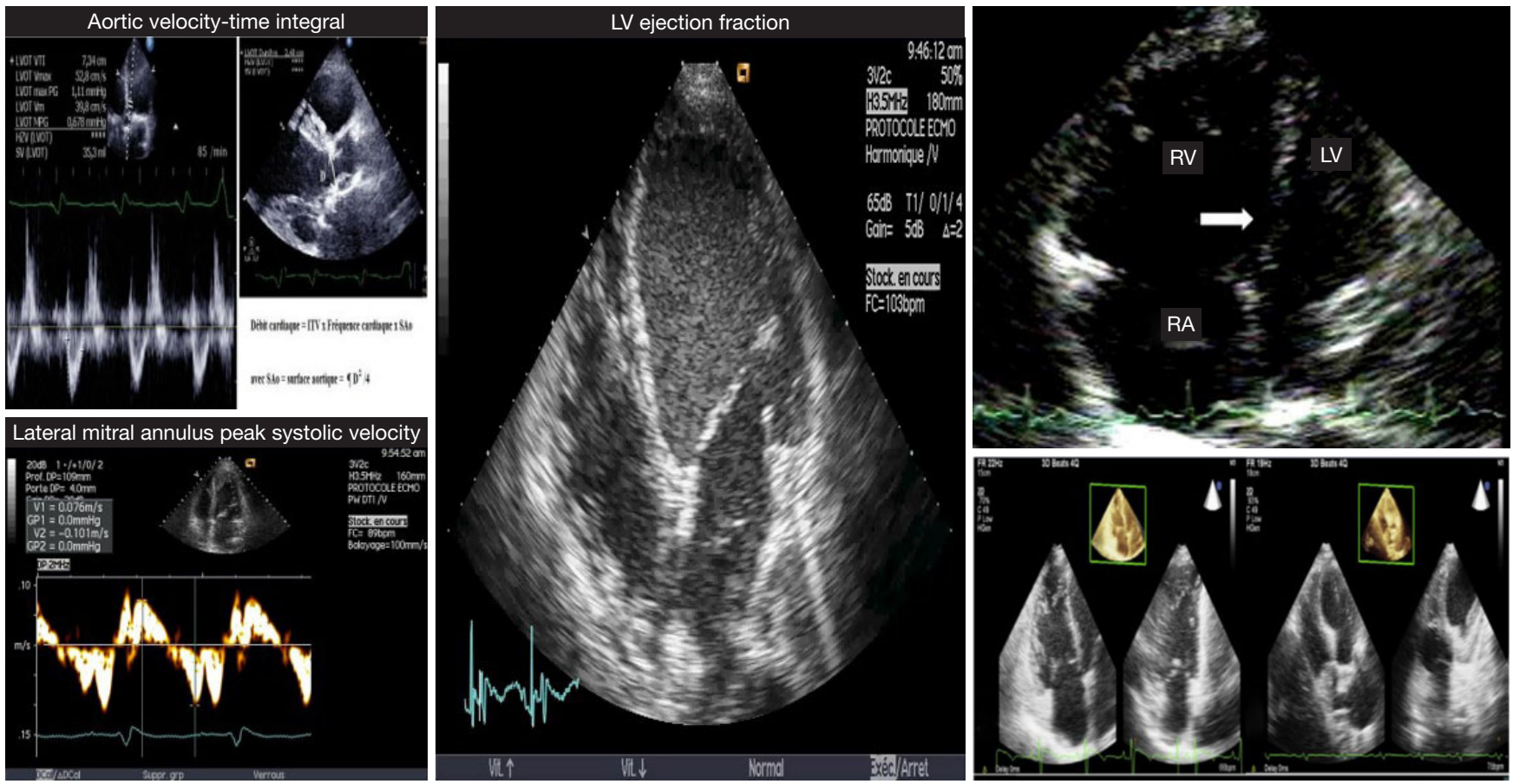

Figure 1 Useful echocardiographic parameters. LV, left ventricle; RV, right ventricle; RA, right atrium.

and technical limitations.

\section{Which conditions are required to attempt weaning from ECMO?}

It is unusual to attempt weaning in the first 72 hours after VA ECMO implantation, because damaged organs need time to recover. However, the duration of ECMO may be shorter in cases of drug intoxication and VA ECMO weaning can be attempted earlier. In most previous studies, the mean duration of support was at least of $3.3 \pm 2.9$ days and was even $8.0 \pm 6.0$ days in one study $(13,26)$. This duration is also necessary to allow the recovery of a potentially "stunned" myocardium $(16,27)$.

Other considerations include the etiology of cardiocirculatory dysfunction, which must be compatible with myocardial recovery (i.e., acute myocarditis, acute myocardial infarction, post-cardiotomy, drug intoxication, septic cardiomyopathy, arrhythmia-induced cardiomyopathy) $(1-5,28)$.

Of note, VA ECMO should not be removed if the patient has not recovered from the conditions which necessitated its implantation (high volume overload and high doses of inotropic agents) (16).

According to the Extracorporeal Life Support
Organization (ELSO) guidelines, hepatic function should have recovered prior to any attempt to wean patients from ECMO, irrespective of the findings of cardiac assessment $(16,29)$. But it does not seem necessary to wait for the recovery of renal function $(26,27,29,30)$.

Furthermore, pulmonary oxygenation of the blood must not be compromised $(3,16,29,31)$. The $\mathrm{PaO}_{2} / \mathrm{FiO}_{2}$ ratio should be more than 200 and the oxygen fraction delivered by the extracorporeal circuit should be $21 \%$ and that delivered by the ventilator circuits should be less than $60 \%$. These measurements should be made 10 minutes after having decreased the ECMO flow and the sweep gas flow, that is, with an ECMO flow rate of less than $1 \mathrm{~L} /$ minute and a sweep gas flow rate of $1 \mathrm{~L} /$ minute for 10 minutes (31).

The patient should be considered hemodynamically stable, that is, they should have a baseline mean arterial pressure (MAP) of $>60 \mathrm{mmHg}$ in the absence or at low doses of vasoactive agents and a pulsatile arterial waveform maintained for at least 24 hours $(13,15,16,29)$.

\section{Why perform weaning trials?}

Weaning trials are essential to assess the behavior of ventricles during increases in preload and to determine whether the ECMO can be safely removed $(14,16,32)$. Load 
conditions can be modified by varying the flow of the VA ECMO centrifugal pump. When ECMO flow is decreased, left ventricle $(\mathrm{LV})$ preload is increased and afterload is decreased (32).

During the decrease of the ECMO flow, the behaviour of the LV and the Frank-Starling reserve can be assessed (32). In 22 refractory cardiogenic shock patients receiving VA ECMO, we varied ECMO flow and examined hemodynamic and echo variables of the failed $\mathrm{LV}$. The initial support was $4.0 \mathrm{~L} / \mathrm{min}$ (IQR, 3.0-4.5 L/min) and could be decreased by a mean of $83 \%$ (32). With this approach, we found significant variations between patients who were successfully weaned and those who were not. The presence of a contractile reserve-a significant increase in LVEF-was associated with successful weaning (32).

This weaning trial is also very important to evaluate right ventricular (RV) function. It is difficult to determine RV function in maximal ECMO flow because the ECMO circuit creates negative pressure and drains venous blood from the right atrium $(13,14,32)$. A reduction in ECMO flow leads to an increase in preload and enables RV function to be assessed $(14,33)$. Thus, in VA ECMO patients, we analyzed the occurrence of a right-left ventricular interdependence defined as the dysfunction of one ventricle secondary to a disorder of the other (33). In refractory CS patients assisted by VA ECMO, we reported an abnormal decrease of the $\mathrm{LV}$ dimensions in the presence of a RV dilatation induced by an increased preload in 33 patients. This interdependence had a strong negative prognostic value, but the study was limited by the small number of patients included and the fact that it came from a single center (33).

Cavarocchi et al. also assessed a weaning strategy with staged decrease of ECMO flow, volume loading and inotrope support over a period of 4 to 6 hours, in 21 patients using transesophageal echocardiography (14). If a ventricle distension occurred, the trial was stopped and the patient was not weaned. The positive predictive value for ventricular recovery was $100 \%$ (95\% CI, $73-100 \%)$.

\section{Which strategies for carrying out ECMO weaning trials?}

Two echocardiographic strategies for carrying out an ECMO weaning trial have been reported in the literature: the first strategy involves trans-thoracic echocardiography (TTE) (13) and the second involves hemodynamic transesophageal echocardiography (hTEE) (14).
In the TTE study, an ECMO weaning trial was undertaken daily if: (I) the patient was considered hemodynamically stable (i.e., baseline mean blood pressure of $>60 \mathrm{mmHg}$ in the absence or at low doses of vasoactive agents and pulsatile arterial waveform maintained for at least $24 \mathrm{~h}$ ) and; (II) pulmonary oxygenation of the blood was not compromised (13). The ECMO flow was decreased to $66 \%$ of the initial flow rate for $10-15 \mathrm{~min}$. It was then decreased to $33 \%$ for $10-15 \mathrm{~min}$ and then to a minimum of $1-1.5 \mathrm{~L} / \mathrm{min}$ for another $10-15 \mathrm{~min}$. If mean blood pressure dropped significantly and was constantly $<60 \mathrm{mmHg}$ during the trial, ECMO flow was returned to $100 \%$ of the initial flow and the trial was stopped. Doppler echocardiography was repeated at each ECMO flow rate. The removal of ECMO was considered if the patient had no end-stage cardiac disease, had partially or fully recovered from the initial cardiac dysfunction, tolerated the full weaning trial and had a LVEF of $>20-25 \%$ and aortic VTI of $>10 \mathrm{~cm}$ under minimal ECMO support.

In the hTEE study, the weaning trial consisted of four stages (14). In the first stage, the authors assessed baseline $\mathrm{LV}$ and RV volume and functions on full-flow ECMO support. During the second stage, ECMO flow was gradually decreased in increments of $0.5 \mathrm{~L} / \mathrm{min}$ to half of the original flow rate (stage 2). Throughout the weaning protocol, hemodynamic responses (i.e., heart rate and blood pressure), RV and LV volumes and functions were monitored continuously. If significant hypotension or $\mathrm{LV}$ or $\mathrm{RV}$ distension occurred, the weaning trial was stopped and the ECMO support was returned to full flow.

Stage 3 consisted of monitoring hemodynamic responses during both volume challenge and a reduction of ECMO flow to a minimum rate of 1.2 to $1.5 \mathrm{~L} / \mathrm{min}$. Volume loading with $5 \%$ albumin $(10 \mathrm{~mL} / \mathrm{kg})$ was used to achieve an appropriate preload. During the last stage (stage 4), $\mathrm{LV}$ and $\mathrm{RV}$ function was assessed during the infusion of inodilators_-dobutamine and/or milrinone-for a period of 4 to 6 hours. These drugs were used to assess RV function in patients with $L V$ dysfunction under consideration for LVAD placement. Final removal of the ECMO was considered if both $\mathrm{LV}$ and $\mathrm{RV}$ functions recovered. If LV dysfunction persisted without RV failure, LVAD implantation was considered.

Of note, most studies suffered from a number of obvious limitations, including small sample size and only providing data from single centers $(13-15,18)$. They also evaluated a mixed population of patients, who had received peripheral and/or central ECMO support, following medical, 


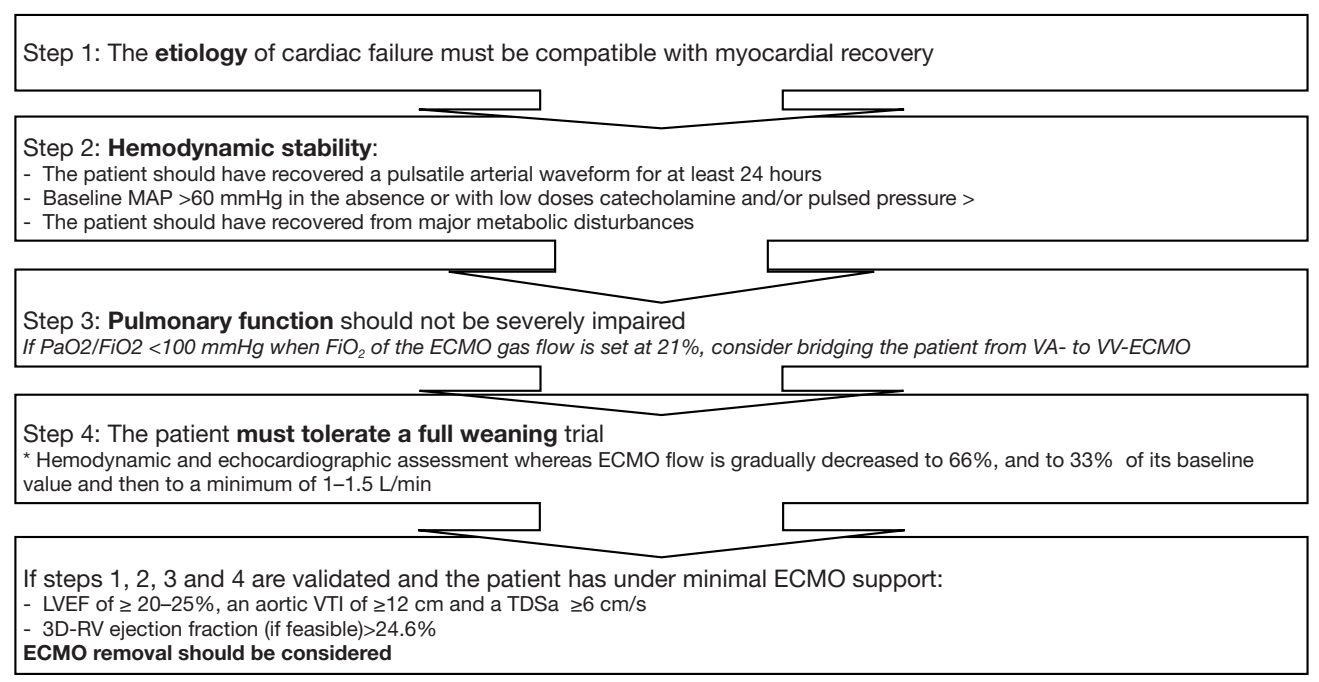

Figure 2 Proposed algorithm for successful weaning from VA ECMO. MAP, mean arterial pressure; VTI, velocity-time integration; LVEF, left ventricular ejection fraction; TDSa, Tissue Doppler lateral mitral annulus peak systolic velocity; RV, right ventricle; CI, cardiac index; PCWP, pulmonary capillary wedge pressure; CVP, central venous pressure.

postcardiotomy, or post-transplantation cardiogenic shock (13-15,18). Some studies were retrospective, and the initiation of decannulation of VA-ECMO was protocoldriven and determined by attending surgeons, which cannot exclude residual confounding and selection bias in the comparison between success and failure (18).

\section{Aids to optimize weaning}

The ability of some medications to facilitate weaning from VA ECMO has been assessed by some $(34,35)$. Levosimendan was assessed in 6 VA ECMO patients with the hypothesis that its remaining effects could favour the weaning from ECMO. This inodilator drug was infused in the patients $24 \mathrm{~h}$ before the planned weaning. In this small study, the use of levosimendan was associated with an increased rate of successful weaning (34). A retrospective, observational, single-center study having assessed 240 VA ECMO patients after cardiovascular surgery reported similar results (34). The authors showed an association between levosimendan treatment and not only successful ECMO weaning [adjusted hazard ratio (HR) 0.41 ; 95\% CI, 0.22-0.80; $\mathrm{P}=0.008$ ], but also longterm mortality (adjusted HR 0.64; 95\% CI, 0.42-0.98; $\mathrm{P}=0.04$ ) (35). These strategies were reported for very small populations or in retrospective studies and must be confirmed in larger series.

\section{Proposed weaning strategy}

In light of all these data, we propose a strategy to optimize weaning from VA ECMO (Figure 2).

\section{Conclusions}

Weaning from VA ECMO remains a difficult decision. We propose a strategy to optimize the weaning process. It is especially important that the ECMO is not removed while the patient is still recovering from the conditions that necessitated the VA ECMO implantation. The etiology of cardio-circulatory dysfunction must be compatible with myocardial recovery and the patient should be considered hemodynamically stable. Weaning trials, alongside hemodynamic and echocardiographic assessments, are indispensable in the assessment of the behavior of the ventricles and when determining whether the ECMO can be removed.

\section{Acknowledgements}

The authors thank Professor Nicolas Danchin for reviewing the English of the manuscript.

\section{Footnote}

Conflicts of Interest: N Aissaoui: Astra-Zeneca, Medtronic, 
Thoratec. The other authors have no conflicts of interest to declare.

\section{References}

1. Abrams D, Combes A, Brodie D. Extracorporeal Membrane Oxygenation in Cardiopulmonary Disease in Adults. J Am Coll Cardiol 2014;63:2769-78.

2. Combes A, Brodie D, Chen YS, et al. The ICM research agenda on extracorporeal life support. Intensive Care Med 2017;43:1306-18.

3. Abrams D, Garan AR, Abdelbary A, et al. Position paper for the organization of ECMO programs for cardiac failure in adults. Intensive Care Med 2018;44:717-29.

4. Le Gall A, Follin A, Cholley B, et al. Veno-arterial-ECMO in the intensive care unit: From technical aspects to clinical practice. Anaesth Crit Care Pain Med 2018;37:259-68.

5. Bréchot $\mathrm{N}$, Luyt $\mathrm{CE}$, Schmidt $M$, et al. Venoarterial extracorporeal membrane oxygenation support for refractory cardiovascular dysfunction during severe bacterial septic shock. Crit Care Med 2013;41:1616-26.

6. Luo XJ, Wang W, Hu SS, et al. Extracorporeal membrane oxygenation for treatment of cardiac failure in adult patients. Interact Cardiovasc Thorac Surg 2009;9:296-300.

7. Mebazaa A, Combes A, van Diepen S, et al. Management of cardiogenic shock complicating myocardial infarction. Intensive Care Med 2018. [Epub ahead of print].

8. Rastan AJ, Dege A, Mohr M, et al. Early and late outcomes of 517 consecutive adult patients treated with extracorporeal membrane oxygenation for refractory postcardiotomy cardiogenic shock. J Thorac Cardiovasc Surg 2010;139:302-11, 311.e1.

9. Combes A, Leprince P, Luyt CE, et al. Outcomes and longterm quality-of-life of patients supported by extracorporeal membrane oxygenation for refractory cardiogenic shock. Crit Care Med 2008;36:1404-11.

10. Chen YS, Chao A, Yu HY, et al. Analysis and results of prolonged resuscitation in cardiac arrest patients rescued by extracorporeal membrane oxygenation. J Am Coll Cardiol 2003;41:197-203.

11. Chang WW, Tsai FC, Tsai TY, et al. Predictors of mortality in patients successfully weaned from extracorporeal membrane oxygenation. PLoS One 2012;7:e42687.

12. Aso S, Matsui H, Fushimi K, et al. In-hospital mortality and successful weaning from venoarterial extracorporeal membrane oxygenation: analysis of 5,263 patients using a national inpatient database in Japan. Crit Care 2016;20:80.
13. Aissaoui N, Luyt CE, Leprince P, et al. Predictors of successful extracorporeal membrane oxygenation (ECMO) weaning after assistance for refractory cardiogenic shock. Intensive Care Med 2011;37:1738-45.

14. Cavarocchi NC, Pitcher HT, Yang Q, et al. Weaning of extracorporeal membrane oxygenation using continuous hemodynamic transesophageal echocardiography. J Thorac Cardiovasc Surg 2013;146:1474-9.

15. Pappalardo F, Pieri M, Arnaez Corada B, et al. Timing and Strategy for Weaning From Venoarterial ECMO are Complex Issues. J Cardiothorac Vasc Anesth 2015;29:906-11.

16. Aissaoui N, El-Banayosy A, Combes A. How to wean a patient from veno-arterial extracorporeal membrane oxygenation. Intensive Care Med 2015;41:902-5.

17. Leick J, Liebetrau C, Szardien S, et al. Door-toimplantation time of extracorporeal life support systems predicts mortality in patients with out-of-hospital cardiac arrest. Clin Res Cardiol 2013;102:661-9.

18. Huang KC, Lin LY, Chen YS, et al. Three-Dimensional Echocardiography-Derived Right Ventricular Ejection Fraction Correlates with Success of Decannulation and Prognosis in Patients Stabilized by Venoarterial Extracorporeal Life Support. J Am Soc Echocardiogr 2018;31:169-79.

19. Akin S, Dos Reis Miranda D, Caliskan K, et al. Functional evaluation of sublingual microcirculation indicates successful weaning from VA-ECMO in cardiogenic shock. Crit Care 2017;21:265.

20. Luyt CE, Landivier A, Leprince P, et al. Usefulness of cardiac biomarkers to predict cardiac recovery in patients on extracorporeal membrane oxygenation support for refractory cardiogenic shock. J Crit Care 2012;27:524.e7-14.

21. Park BW, Seo DC, Moon IK, et al. Pulse pressure as a prognostic marker in patients receiving extracorporeal life support. Resuscitation 2013;84:1404-8.

22. Li CL, Wang H, Jia M, et al. The early dynamic behavior of lactate is linked to mortality in postcardiotomy patients with extracorporeal membrane oxygenation support: A retrospective observational study. J Thorac Cardiovasc Surg 2015;149:1445-50.

23. Platts DG, Sedgwick JF, Burstow DJ, et al. The role of echocardiography in the management of patients supported by extracorporeal membrane oxygenation. J Am Soc Echocardiogr 2012;25:131-41.

24. Donker DW, Meuwese CL, Braithwaite SA, et al. Echocardiography in extracorporeal life support: A key 
player in procedural guidance, tailoring and monitoring. Perfusion 2018;33:31-41

25. Aziz TA, Singh G, Popjes E, et al. Initial experience with CentriMag extracorporal membrane oxygenation for support of critically ill patients with refractory cardiogenic shock. J Heart Lung Transplant 2010;29:66-71.

26. Baud FJ, Megarbane B, Deye N, et al. Clinical review: aggressive management and extracorporeal support for drug-induced cardiotoxicity. Crit Care 2007;11:207.

27. Durinka JB, Bogar LJ, Hirose H, et al. End-organ recovery is key to success for extracorporeal membrane oxygenation as a bridge to implantable left ventricular assist device. ASAIO J 2014;60:189-92.

28. Dangers L, Bréchot N, Schmidt M, et al. Extracorporeal Membrane Oxygenation for Acute Decompensated Heart Failure. Crit Care Med 2017;45:1359-66.

29. ELSO Guidelines for Cardiopulmonary Extracorporeal Life Support Extracorporeal Life Support Organization, Version 1.3 November 2013 Ann Arbor, MI, USA. Available online: http://www.elsonet.org

30. Khot UN, Mishra M, Yamani MH, et al. Severe renal dysfunction complicating cardiogenic shock is not a contraindication to mechanical support as a bridge to

Cite this article as: Ortuno S, Delmas C, Diehl JL, Bailleul C, Lancelot A, Naili M, Cholley B, Pirracchio R, Aissaoui $\mathrm{N}$. Weaning from veno-arterial extra-corporeal membrane oxygenation: which strategy to use? Ann Cardiothorac Surg 2019;8(1):E1-E8. doi: 10.21037/acs.2018.08.05 cardiac transplantation. J Am Coll Cardiol 2003;41:381-5.

31. Schmidt M, Pellegrino V, Combes A, et al. Mechanical ventilation during extracorporeal membrane oxygenation. Crit Care 2014;18:203.

32. Aissaoui N, Guerot E, Combes A, et al. Two-dimensional strain rate and Doppler tissue myocardial velocities: analysis by echocardiography of hemodynamic and functional changes of the failed left ventricle during different degrees of extracorporeal life support. J Am Soc Echocardiogr 2012;25:632-40.

33. Aissaoui N, Caudron J, Leprince P, et al. Right-left ventricular interdependence: a promising predictor of successful extracorporeal membrane oxygenation (ECMO) weaning after assistance for refractory cardiogenic shock. Intensive Care Med 2017;43:592-4.

34. Affronti A, di Bella I, Carino D, et al. Levosimendan may improve weaning outcomes in venoarterial ECMO patients. ASAIO J 2013;59:554-7.

35. Distelmaier K, Roth C, Schrutka L, et al. Beneficial effects of levosimendan on survival in patients undergoing extracorporeal membrane oxygenation after cardiovascular surgery. Br J Anaesth 2016;117:52-8. 